\title{
Insulin Potentiation Therapy in the Treatment of Malignant Neoplastic Diseases: A Three Year Study
}

\author{
Christo Damyanov*, Gherasimova DM, Avramov LA and Masley IK
}

Medical Center "Integrative Medicine," Sofia, Bulgaria

\begin{abstract}
Problem Statement: Even after decades of scientific research, the application of chemotherapy in the management of neoplastic disease still presents numerous difficulties. Significant, amongst potential complications are numerous toxicity related side effects and the potential for chemoresistance. Despite the widespread tendency to include a variety of new chemotherapeutics in different combinations, progress in this area has proven slow going and unsatisfactory due to the aforementioned factors.
\end{abstract}

Approach: Seeking a new approach, we introduced the method of Insulin Potentiation Therapy (IPT) in our practice. The theoretical basis and the gathered experimental data on insulin's mode of action, as well as its application in practice, show that IPT is a promising method with low toxicity. Moreover, it facilitates a selectively physiological approach to the management of neoplastic disease using chemotherapy.

In this report we present the results of our three-year experience applying Insulin Potentiation Targeted Therapy Low Dose (IPTLD) in the treatment of 196 patients diagnosed with a variety of neoplastic diseases.

Results: Our results showed that patients tolerated IPTLD without difficulties, without serious side effects. Our laboratory tests demonstrated that the dose related toxicity of chemotherapeutics could be largely mitigated when applied in conjunction with insulin, at a fractionated dose in accordance with a dose dense regimen. Upon follow-up, eighty five of 106 patients $(80 \%)$ with advanced metastatic disease reported a subjectively significant improvement in their quality of life.

Conclusions: Future extended experimental data and clinical trials would contribute to a more complete understanding of the therapeutic potential of IPTLD.

Keywords: Oncology; Cancer treatment; Insulin potentiation targeted low dose; Low-dose chemotherapy

\section{Introduction}

A basic principle in the conventional administration of chemotherapy is the application of maximum tolerated doses at extended intervals of time. Despite the development of novel chemotherapeutics and varied combinations in numerous clinical trials, advancement has proven slow going and arduous. Difficulties such as poorly tolerated levels of toxicity, chemo resistance and an absence of significant prolongation of life expectancy are still unresolved. [1-3].

Efforts in the scientific community over the last decade or so, have led to a new strategy in the application of chemotherapeutics. So called "metronomic chemotherapy" involves the use of reduced doses of drugs at shorter intervals of time. Initial experimental and clinical results involving this strategy are promising and prompt further investigation [4-6].

A somewhat similar method in the application of chemotherapeutics likewise involves fractionated doses of conventional chemotherapeutics administered at short intervals. This method departs from the heretofore standard application of chemotherapy with the use of insulin as a biologic response modifier. Commonly referred to as Insulin Potentiation Therapy (IPT) or Insulin Potentiation Targeted Low Dose (IPTLD), this method facilitates the selective targeting of malignant cells while sparing normal tissues, resulting in greatly reduced treatment toxicity and improved quality of life as reported by patients [7-11].

Insulin Potentiation Therapy was developed in 1932 by the Mexican military physician Donato Perez Garcia, who reported anecdotal success in clinical practice over the course of three decades. A proposed theoretical mechanism of action was developed scientifically and presented in two consecutive publications by Ayre et al. $[7,8]$. In the last decade this method has gained in visibility and popularity and is currently practiced by an increasing number of physicians all over the world.

\section{Materials and Methods}

Between March 2006 and October 2009, 196 patients with biopsy confirmed diagnoses received IPTLD in the management of their disease. Eight patients (4\%) were treated adjuvantly following surgical removal of localized tumors and 188 patients presented with advanced metastatic disease. Upon intake, a physical exam was performed and patients were given a detailed description of the IPTLD protocol. All patients gave written consent for the treatments they received. Table 1 outlines the demographic and clinical characteristics of the subjects involved and Table 2 categorizes the primary origin of disease and the number of patients with each tumor location.

*Corresponding author: Christo Damyanov, MD, PhD, bl.330 "Delijska vodenica", "Drujba 2", Medical Center "Integrative Medicine", 1582 Sofia, Bulgaria, Tel: +359 2 44246 87; +359 244247 31; Fax: +359 8861035 96; E-mail: integramed@mail.bg

Received April 04, 2012; Accepted April 23, 2012; Published April 25, 2012

Citation: Damyanov C, Gherasimova DM, Avramov LA, Masley IK (2012) Insulin Potentiation Therapy in the Treatment of Malignant Neoplastic Diseases: A Three Year Study. J Cancer Sci Ther 4: 088-091. doi:10.4172/1948-5956.1000117

Copyright: (c) 2012 Damyanov C, et al. This is an open-access article distributed under the terms of the Creative Commons Attribution License, which permits unrestricted use, distribution, and reproduction in any medium, provided the original author and source are credited. 


\begin{tabular}{|c|c|}
\hline Total number of patients & 196 \\
\hline Age (mean) & 57 \\
\hline Sex, m/fm ratio & 1.32 \\
\hline Karnofsky Performance Scale (mean) & 69 \\
\hline Preceding Surgical treatment & 108 \\
\hline Preceding Hormonal Therapy & 11 \\
\hline Preceding Chemotherapy & 36 \\
\hline Preceding Radiotherapy & 16 \\
\hline Stage I & 8 \\
\hline Stage II & 14 \\
\hline Stage III & 33 \\
\hline Stage IV & 141 \\
\hline
\end{tabular}

Table 1: Clinical characteristics of involved subjects.

\begin{tabular}{|c|c|}
\hline Lungs & 30 \\
\hline Mammary gland & 27 \\
\hline Prostate gland & 18 \\
\hline Stomach & 21 \\
\hline Urinary bladder & 12 \\
\hline Large intestine & 8 \\
\hline Ovaries & 13 \\
\hline Pancreas & 10 \\
\hline Uterus & 9 \\
\hline Liver & 3 \\
\hline Malignant melanoma & 4 \\
\hline Head and Neck & 11 \\
\hline Others & 30 \\
\hline
\end{tabular}

Table 2: Location of the tumor and number of the affected patients.

Prior to the initiation of therapy, standard clinical data was collected for each subject. Imaging studies were performed at different medical institutions, namely CT scans, MRI, ultrasound, bone scintigraphy, and chest X-rays. Laboratory tests included complete blood count, serum albumin, blood sugar, urea, creatinine, cholesterol, blood mineral balance, liver tests, coagulation status, CRP, urine samples, tumor markers and ferritin levels. Control lab tests, including tumor markers, were performed following the sixth IPTLD treatment and every subsequent fourth treatment. Control imaging studies were performed after the tenth procedure and follow-up imaging was done at three and sixth months after the initiation of treatment. When needed, control lab tests were run after the third procedure. The patients'status prior to the initiation of treatment was measured using the Karnofski Performance Status scale. Upon intake and once a month thereafter, patients completed the Beretta Self Compilation Questionnaire to evaluate their subjective status [12]. It was used as a follow-up evaluation of the patients' status calculating the results from sections A and B of the questionnaire. Figure 1 describes Beretta Self Compilation Questionnaire.

Short acting insulin (0.4 units/kg body weight) was used in conjunction with various cytostatic drug regimens. Standard chemotherapy regimens were used (as developed) in a clinical trial setting according to diagnosis; however, the dose administered was approximately one tenth of that given in a conventional setting. When patients had received standard dose chemotherapy prior to initiating IPTLD, we initiated different chemotherapeutic combinations to reduce the possibility of chemoresistance. Alternating chemotherapy protocols were employed in patients whose disease demonstrated marked progression. Our course of treatment consisted of 6 IPTLD treatments, one per week for six consecutive weeks. At the conclusion of that six-week time period, we conducted lab tests to evaluate the patients' condition. In patients who experienced a favorable response, we continued with another four IPTLD treatments, at intervals of one treatment every ten days. Follow-up or maintenance treatments were conducted at a frequency of one treatment every two weeks, with the frequency gradually decreasing to once every three or more weeks between treatments.

Ancillary medications taken concurrently include hepatoprotectors, angiogenesis inhibitors, dexamethasone, COX-2 inhibitors, and low doses of oral cyclophosphamide. Ancillary therapies incorporated as part of a comprehensive approach to disease management included dietary modifications, caffeine enemas, oral and intravenous antioxidants, ozone therapy, and biophotonic therapy [13].

Patient management removed from the strictly controlled context of a clinical trial can lead to a myriad of practical challenges, including incomplete imaging and lab studies and patients who discontinue further treatment. Consequently, our criteria in determining and assessing treatment efficacy is defined by the number of subjectively improved patients, laboratory results including tumor markers, the incidence and severity of side-effects, and overall quality of life as selfreported by the patient.

\section{Results}

Forty eight of one hundred ninety six patients (24.4\%) discontinued treatment before the sixth IPTLD treatment. Financial constraints numbered among the reasons for this occurrence. Twenty one of one hundred ninety six patients (10.7\%) discontinued treatment before the tenth procedure. The average number of IPTLD treatments received amongst patents who completed the initial six was thirteen treatments total.

Our patients reported tolerating IPTLD with few difficulties. Side effects reported included general fatigue and lethargy on the day of treatment. Four of the one hundred forty-eight patients who had more than six IPTLD procedures (1.3\%) reported nausea and vomiting upon receiving treatment. Patients reported these side effects resolving the following day. Two of 148 patients (1.36\%) demonstrated an allergic reaction to vitamin $\mathrm{B}$ complex, which was easily controlled by an application of corticosteroids.

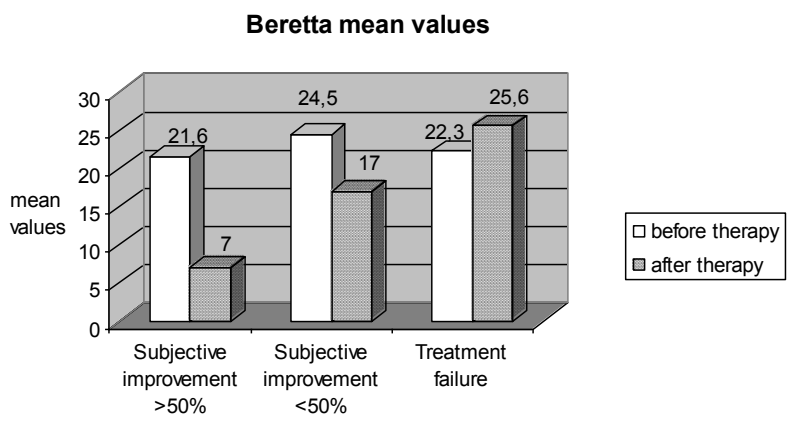

Figure 1: Mean values of Beretta symptomatic index results. 
Patients with normal blood counts upon the initiation of treatment did not experience any significant changes. Hemoglobin $(\mathrm{Hb})$ levels slightly decreased in sixty five $(43.9 \%)$ of one hundred forty eight patients. Only two of the 148 patients with initially low $\mathrm{Hb}$ level needed blood transfusion while in active treatment. Liver enzymes were elevated slightly and temporarily in twenty-three (26\%) of eighty-six patients with normal liver tests at intake. This condition did not affect the patients' subjective status. These variations in $\mathrm{Hb}$ and liver enzymes rate a Grade 0 as measured by the Eastern Cooperative Oncology Group (ECOG) Toxicity Criteria scale [14].

Four of 148 patients with chronic renal failure demonstrated increased levels of serum creatinine, however, this condition did not interfere with their treatment schedule. None of these four patients discontinued therapy or required ameliorative intervention in the management of their renal failure.

None of the eight patients treated adjuvantly following the surgical removal of localized tumors developed measurable disease during a mean follow-up period of 11 months. One patient with a diagnosis of breast cancer did experience a reoccurrence of her disease. She had initially undergone an operation for a $6 \mathrm{~cm}$ lesion and subsequently received 15 IPTLD applications in an adjuvant setting. Imaging tests at the conclusion of those 15 treatments did not detect measurable disease. Nineteen months following the conclusion of her course of IPTLD treatments a subsequent surgery revealed a residual tumor of $1.5 \mathrm{~cm}$. While the experience of these eight patients is of anecdotal interest, the small, statistically insignificant number of patients and the short follow-up period does not lead to substantiated conclusions concerning the effectiveness of IPTLD as treatment in such cases.

One measure of therapy response we employed in one hundred six patients was the Beretta Symptomatic Index. Figure 2 describes a compilation of the Beretta symptomatic index results with the mean values of three groups of patients. Thirty patients out of 106 $(28 \%)$ reported improvement in their subjective status by more than $50 \%$ (group 1). Fifty five patients (51.8\%) described a subjective improvement of less than $50 \%$ (group 2). Twenty one patients (19.8\%) reported deterioration or no significant symptomatic index change between the initiation and conclusion of their course of IPTLD treatments (group 3).

A reduction in tumor markers was observed in forty five (53\%) of eighty five patients who completed six treatments. Tumor markers in seven $(8 \%)$ of those eighty five measured within range after six treatments.

Nine patients presented with type II diabetes. Over the course of treatment, the blood sugar levels in eight of these patients normalized (89\%).

\section{Discussion}

Sixty percent of patients with metastatic disease reported a subjective improvement in the quality of their life upon receiving their first treatment. Eighty five of one hundred six patients treated (80\%) reported a significant improvement in the quality of their life upon the completion of six treatments. The longest period of disease remission we measured was in a 60-year-old female patient who had undergone an operation for gastric cancer. She was diagnosed with metastatic disease upon discovery of secondary lesions in her liver. After receiving one standard dose chemotherapy treatment, the patient discontinued therapy due to side effects. She was enrolled in our program and

\section{Self Compilation Questionnaire for Weekly Determination of Subjective Status} Table 46

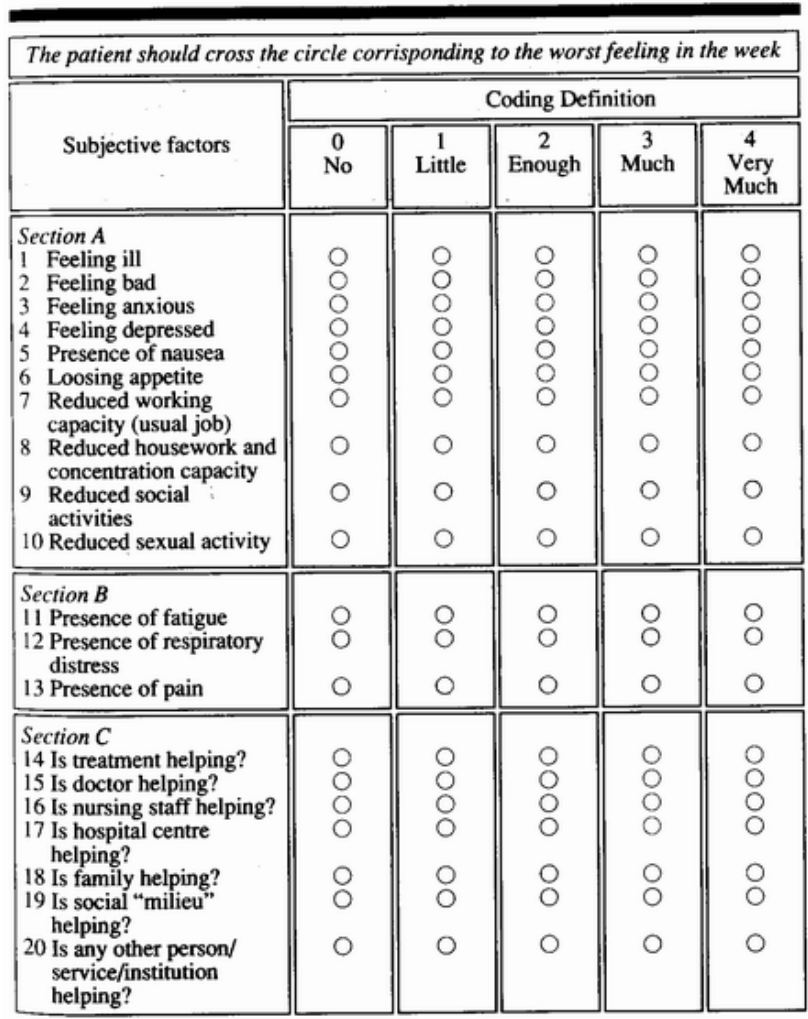

Beretta G. - Cancer Chemother. Regimens, 1986, 9, 37

Figure 2: Beretta Self Compilation Questionnaire.

underwent 10 IPTLD treatments. Imaging studies did not reveal any evidence of disease and lab tests revealed tumor markers within normal range. At present, the patient continues free of all evidence of disease, capable to work without hindrance, and reports an excellent quality of life. Three out of 106 patients with advanced metastatic disease experienced remission from their disease for a period of twenty four months or more.

A variety of factors come into play in determining what can be considered a favorable response to IPTLD treatment, such as the extent of disease upon initiation of treatment and whether the patient had previously received standard dose chemotherapy or radiotherapy. Our findings on the application of IPTLD in patients with advanced metastatic disease who have failed both standard dose chemotherapy and radiotherapy can be found in a separate publication. In that publication, we present three patient case studies that demonstrate the capacity of IPTLD as an effective palliative measure that can be applied once the standard of care has failed. One such case describes a 56 year old patient treated for advanced metastatic hormone-refractory prostate cancer who experienced a complete remission of disease for a follow-up period of 20 months [6].

There exists a gap in standard oncological practice in the satisfactory management and palliation of the pain and discomfort that can accompany malignant disease processes in patients for whom 
Citation: Damyanov C, Gherasimova DM, Avramov LA, Masley IK (2012) Insulin Potentiation Therapy in the Treatment of Malignant Neoplastic Diseases: A Three Year Study. J Cancer Sci Ther 4: 088-091. doi:10.4172/1948-5956.1000117

the standard of care has failed. IPTLD represents a viable option for such patients, effectively filling a gap in oncological care for a form of treatment that simultaneously palliates symptoms and provides for the possibility, in some cases, of reversing or resolving the malignant process.

Insulin Potentiation Therapy can be safely employed with diabetic patients. The treatment is easily tolerated, and can be carried out in the absence of detrimental complications or side-effects. In two patients with type II diabetes, their usual morning insulin dosage was applied before their IPTLD treatment. During the treatment 8 out of 9 diabetic patients had permanently lower blood sugar levels.

Patients easily tolerated IPTLD. No serious side effects or complications were reported, with the exception of two patients with pre/existing anemic syndrome, who eventually required blood transfusions. The most commonly reported effects of treatment were fatigue and lethargy on the day of treatment, and these effects did not disrupt treatment or require additional medications. These side effects resolved the day following treatment. Lab control tests did not reveal any significant toxicity, as defined by ECOG common toxicity criteria. Of additional interest, IPTLD can be employed in the care of patients with serious pre/existing conditions or patients who are not eligible to receive standard dose chemotherapy due to concerns of safety.

We are constantly seeking to improve IPTLD efficacy and outcomes, as well as develop an effective maintenance strategy for patients who have achieved a remission from their disease. The results we obtained in the application of IPTLD in the management of advanced, chemotherapy refractive metastatic disease as an effective means of palliating the pain and discomfort assoicated with the malignant disease processes proved exceptional.

\section{Conclusion}

Combining the available theoretical data gathered on the use of insulin as a biologic response modifier with the experiences of a growing number of physicians who employ the method, as well as on our own humble experience, we sincerely believe that the method presents a compelling opportunity for solving the problem with chemotherapy's toxicity, for improving treatment effectiveness and quality of life. The results we achieved, applying IPTLD, and most importantly its lower toxicity, enabled us to apply it as a leading method in the therapy of cancer patients in our practice.

Future extended experimental data and clinical trials would contribute to a more complete understanding of the therapeutic potential of IPTLD, and its capacity to play a role as a valuable treatment modality in the complex therapy of neoplastic disease, where applicable and appropriate.

The authors of this article have no conflicts of interest to disclose. There are no personal or professional relationships that would inappropriately influence this work.

\section{References}

1. Millar AW, Lynch KP (2003) Rethinking clinical trials for cytostatic drugs. Nat Rev Cancer 3: 540-545.

2. Hanahan D, Bergers G, Bergsland E (2000) Less is more, regularly: metronomic dosing of cytotoxic drugs can target tumor angiogenesis in mice. J Clin Invest 105: 1045-1047.
3. Kerbel RS, Klement G, Pritchard KI, Kamen B (2002) Continuous low-dose anti-angiogenic/ metronomic chemotherapy: from the research laboratory into the oncology clinic. Ann Oncol 13: 12-15.

4. Bocci G, Tuccori M, Emmenegger U, Liguori V, Falcone A, et al (2005) Cyclophosphamide-methotrexate 'metronomic' chemotherapy for the palliative treatment of metastatic breast cancer. A comparative pharmacoeconomic evaluation. Ann Oncol 16: 1243-1252.

5. Fidler IJ, Ellis LM (2000) Chemotherapeutic drugs--more really is not better Nat Med 6: 500-502.

6. Damyanov C, Radoslavova M, Gavrilov V, Stoeva D (2009) Low dose chemotherapy in combination with insulin for the treatment of advanced metastatic tumors. Preliminary experience. J BUON 14: 711-715.

7. Ayre SG, Perez Garcia y Bellon D, Perez Garcia D Jr (1986) Insulin potentiation therapy: a new concept in the management of chronic degenerative disease. Med Hypotheses 20: 199-210.

8. Ayre SG, Garcia y Bellon DP, Garcia DP Jr (2000) Insulin, chemotherapy, and the mechanisms of malignancy: the design and the demise of cancer. Med Hypotheses 55: 330-334.

9. Damyanov C, Radoslavova M, Gavrilov V (2008) The Insulin Potentiated Therapy (IPT) in the Treatment of Chronic and Oncological Diseases. Journal MED info 11: 55-59.

10. Hauser RA (2002) Treating cancer with insulin potentiation therapy. Beulah Land Press, Oak Park, Illinois.

11. Lasalvia-Prisco E, Cucchi S, Vázquez J, Lasalvia-Galante E, Golomar W, et al., (2004) Insulin-induced enhancement of antitumoral response to methotrexate in breast cancer patients. Cancer Chemother Pharmacol 53: 220-224.

12. Beretta GD (1991) Cancer treatment medical guide. (10 Edn), Farmitalia Carlo Erba-Erbamont, Milan, Italy.

13. Bocci V, Larini A, Micheli V (2005) Restoration of normoxia by ozone therapy may control neoplastic growth: a review and a working hypothesis. J Altern Complement Med 11: 257-265.

14. Oken MM, Creech RH, Tormey DC, Horton J, Davis TE, et al. (1982) Toxicity and response criteria of the Eastern Cooperative Oncology Group. Am J Clin Oncol 5: 649-655. 